\title{
Pressure dependence of optical transitions in semiconducting single-walled carbon nanotubes
}

\author{
W. Shan ${ }^{*}, 1$, J. Wu ${ }^{1}$, W. Walukiewicz ${ }^{1}$, J. W. Ager III ${ }^{1}$, K. M. Yu ${ }^{1}$, E. E. Haller ${ }^{1,2}$, \\ K. Kissell ${ }^{3}$, S. M. Bachilo ${ }^{3}$, R. B.Weisman ${ }^{3}$, and R. E. Smalley ${ }^{3}$ \\ ${ }^{1}$ Materials Sciences Division, Lawrence Berkeley National Laboratory, Berkeley, CA 94720, USA \\ 2 Department of Materials Science and Engineering, University of California, Berkeley, CA 94720, USA \\ ${ }^{3}$ Department of Chemistry, Rice University, 6100 Main St., Houston, TX 77005, USA
}

Received 31 July 2004, revised 22 August 2004, accepted 14 September 2004

Published online 27 October 2004

PACS 62.25.+g, 62.50.+p, 71.20.Tx, 78.67.Ch

The effects of applied hydrostatic pressure on the optical transitions in semiconducting single-walled carbon nanotubes with different diameters and chiralities have been studied using optical absorption and photoluminescence spectroscopy. The excitonic transitions associated with the band-gap (the first van Hove transition) energies in the carbon nanotubes are found to shift to lower energy at a rate depending on nanotube structure. The excitonic transitions between the first excited confinement states (the second van Hove transitions) are found to be much less sensitive to the applied hydrostatic pressure. All nanotubes show a dependence on their chirality in terms of pressure coefficient of the band-gap energy, with the larger-diameter nanotubes exhibiting a higher sensitivity of the band-gap energy to hydrostatic pressure.

(C) 2004 WILEY-VCH Verlag GmbH \& Co. KGaA, Weinheim

\section{Introduction}

The size-dependent quantum confinement effects in semiconducting nanostructures such as quantum wires and quantum dots have generated tremendous research interest. The confinement effects result in drastic changes in the electronic properties and provide great potential for new technological applications. The unique electronic and mechanical properties of carbon nanotubes have attracted much attention in recent years. The properties of carbon nanotubes are determined by their diameter and chiral angle. The nanotubes can be either metallic or semiconducting depending on their chiral structure indexed by two integers $(n, m)$ [1]. It has also been found that the single-walled nanotubes (SWNTs) have superior elastic properties capable of accommodating large mechanical strains [2]. Recently, it has been predicted theoretically that under hydrostatic compression conditions the band gap of a semiconducting SWNT should increase or decrease depending on whether the remainder of dividing $n-m$ by 3 , i.e. $q=(n-m) \bmod 3$, is equal to 1 or 2 [3]. In this work, we report our recent pressure dependent studies on the optical transitions between the first van Hove singularities (the band gaps) and the second van Hove singularities below and above the Fermi level in individual single-walled carbon nanotubes. By examining the optical transitions under pressure using optical absorption and photoluminescence measurements, the pressure coefficients of the band-gap energies for individual SWNTs under hydrostatic conditions were determined. Our results show that the pressure coefficients obtained from the nanotubes with various structural configurations do depend on nanotube structures but do not increase with pressure. Furthermore, the variation of the pressure coefficients can be scaled with nanotube structures in terms of tube diameter and chirality.

\footnotetext{
* Corresponding author: e-mail: WShan@|bl.gov
} 


\section{Experimental}

Single-walled carbon nanotubes grown in high-pressure carbon monoxide are suspended by aqueous sodium dodecyl sulfate (SDS) surfactant in deuterium oxide $\left(\mathrm{D}_{2} \mathrm{O}\right)$ and treated by intensive ultrasonication and subsequent centrifugation [4]. The resultant suspension is rich in dispersed individual SWNTs that are in van der Waals contact with the surrounding SDS surfactant coating, which is presumed to reduce the fluorescence quenching effects by isolating semiconducting nanotubes from metallic ones. AFM shows that the nanotube length is about $150 \mathrm{~nm}$ as a result of the ultrasonication cutting.

Optical absorption and photoluminescence (PL) measurements were performed at room temperature. The light source for absorption measurement is a halogen tungsten lamp dispersed by a 0.5 m monochromator. A Ge or Si photodiode is used as detector depending on the desired measurement wavelength range. For PL measurements, either an $\mathrm{Ar}^{+}$laser $(476.5 \mathrm{~nm})$ or a $\mathrm{HeCd}$ laser $(325 \mathrm{~nm})$ is used as the excitation source and a 1-m double-grating monochromator is used to disperse the PL signals. Carbon nanotube suspension is sealed directly in diamond anvil cell. The standard ruby R1 line method is used for pressure calibration.

\section{Results and discussion}

Figure 1 shows the absorption spectrum of the samples at ambient. The observed absorption peaks can be divided into two groups. In the energy region below $\sim 1.4 \mathrm{eV}, 12$ absorption peaks (Group A transitions) are observed with full width at half maximum (FWHM) between $10 \mathrm{meV}$ and $30 \mathrm{meV}$. In the energy region between $1.4 \mathrm{eV}$ and $3.1 \mathrm{eV}$, there is another set of twelve peaks (Group B transitions) with FWHM between $50 \mathrm{meV}$ and $100 \mathrm{meV}$. The different characteristics between these two groups are attributed to the different pairs of energy levels involved in the optical transitions. Group A peaks correspond to optical transitions across the band gaps of the semiconducting nanotubes between the lowest unoccupied van Hove singularity (VHS) and the highest occupied VHS, and are conventionally denoted as $E_{11}$. Group B peaks $\left(E_{22}\right)$ are attributed to the transitions between the second pair of VHS above and below the Fermi level, respectively [5]. $\mathrm{E}_{22}$ peaks are much wider than the thermal broadening energy due to lifetime broadening. These assignments can be experimentally verified by PL measurements. Photo-excitation of the samples generates strong emission in the spectral range of Group A transitions, as shown in the inset of the figure, with each PL peak position well coinciding with a corresponding absorption peak. In contrast, as expected, no such characteristic emission can be detected in the spectral range of Group B transitions (data not shown here).

Each observed optical transition can be assigned to a specific SWNT structure with a pair of structure indices $(n, m)$ based on an excitation-emission spectrofluoimetric mapping reported by Bachilo et al. [6].

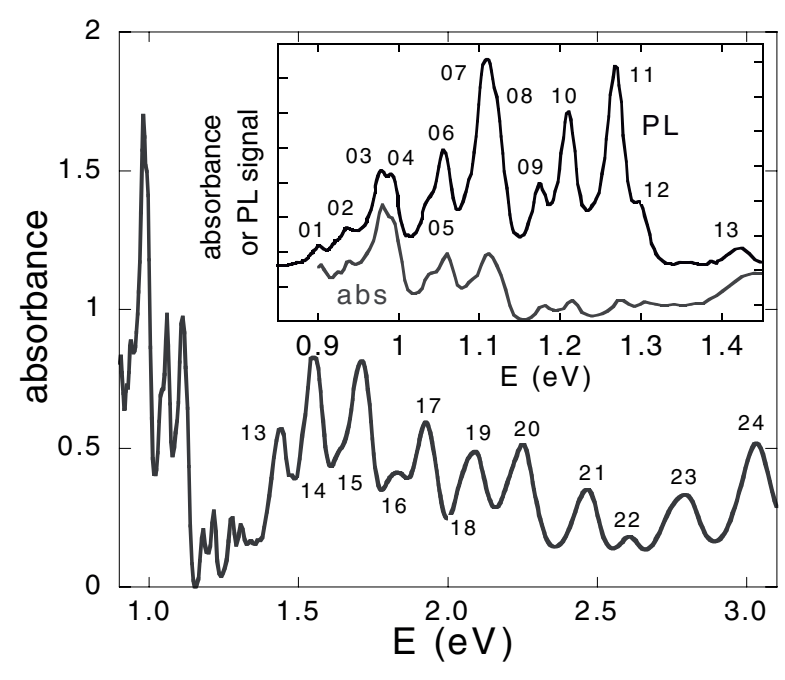

Fig. 1 Absorption spectrum recorded from the SWNT suspension used in this study. The inset shows the comparison between absorption and PL spectra in the near infrared spectral region. 
Table 1 Absorption peak energies, linear pressure coefficients, deformation potentials and corresponding nanotube assignments.

\begin{tabular}{rlllclll}
\hline $\begin{array}{l}E_{11} \\
\text { peak }\end{array}$ & $\begin{array}{l}E_{11} \\
(\mathrm{eV})\end{array}$ & $\begin{array}{l}\mathrm{d} E_{11} / \mathrm{d} P \\
(\mathrm{meV} / \mathrm{kbar})\end{array}$ & $\begin{array}{l}\mathrm{d} E_{11} / \mathrm{d} \ln \mathrm{V} \\
(\mathrm{eV})\end{array}$ & $\begin{array}{l}\text { Assignment } \\
(n, m)\end{array}$ & $\begin{array}{l}E_{22} \\
\mathrm{peak}\end{array}$ & $\begin{array}{l}E_{22} \\
(\mathrm{eV})\end{array}$ & $\begin{array}{l}\mathrm{d} E_{22} / \mathrm{d} P \\
(\mathrm{meV} / \mathrm{kbar})\end{array}$ \\
\hline 1 & 0.899 & & & $(9,8)$ & 14 & 1.543 & -0.3 \\
2 & 0.933 & & & $(12,2)$ & 16 & 1.828 & \\
3 & 0.977 & -2.9 & 6.4 & $(8,7)$ & 15 & 1.700 & -1.3 \\
4 & 0.994 & & & $(11,1)$ & 19 & 2.083 & -0.7 \\
5 & 1.035 & -4.6 & 10.3 & $(11,3)$ & 14 & & \\
6 & 1.054 & -3.0 & 7.0 & $(8,6)$ & 15 & & \\
7 & 1.105 & -2.0 & 4.9 & $(7,6)$ & 17 & 1.917 & -1.6 \\
8 & 1.123 & & & $(9,4)$ & 15 & & \\
9 & 1.173 & -3.8 & 9.5 & $(10,2)$ & 15 & & \\
10 & 1.209 & -1.8 & 4.7 & $(7,5)$ & 17 & & \\
11 & 1.272 & -1.0 & 2.8 & $(6,5)$ & 20 & 2.239 & -0.4 \\
12 & 1.295 & & & $(8,3)$ & 17 & & \\
13 & 1.428 & -0.6 & 2.1 & $(6,2)$ & 24 & 3.030 & \\
\hline
\end{tabular}

The equation that approximately relates the nanotube geometry to the optical transition energies is given by

$$
E_{i i}=\frac{A_{i i}}{B_{i i}+d}+\frac{C_{i i} \cdot \cos 3 \alpha}{d^{2}}
$$

where $i=1$ or $2, d$ and $\alpha$ are the nanotube diameter and the chiral angle, respectively. $A_{i i}, B_{i i}$ and $C_{i i}$ are empirical constants given in Ref. [6]. The sign and the numerical value of $C_{i i}$ also depend on the value of $q=(n-m) \bmod 3$. Every peak in Groups A and B is assigned a pair of structure indices $(n, m)$ using Eq. (1). The assignment is listed in Table 1 . The fact that several $E_{11}$ peaks correspond to the same $E_{22}$ peak indicates that the $E_{22}$ peak includes contributions from several different nanotube structures. Peaks 18, 21, 22 and 23 do not match any $E_{11}$ peaks observed in the spectral range. They are attributed to the lowest-energy van Hove transitions of metallic nanotubes in the sample [6].

The effect of hydrostatic pressure on the absorption spectrum of Group A peaks is shown in the left panel in Fig. 2. The nearly equal-distanced oscillatory noises on some absorption curves are interference
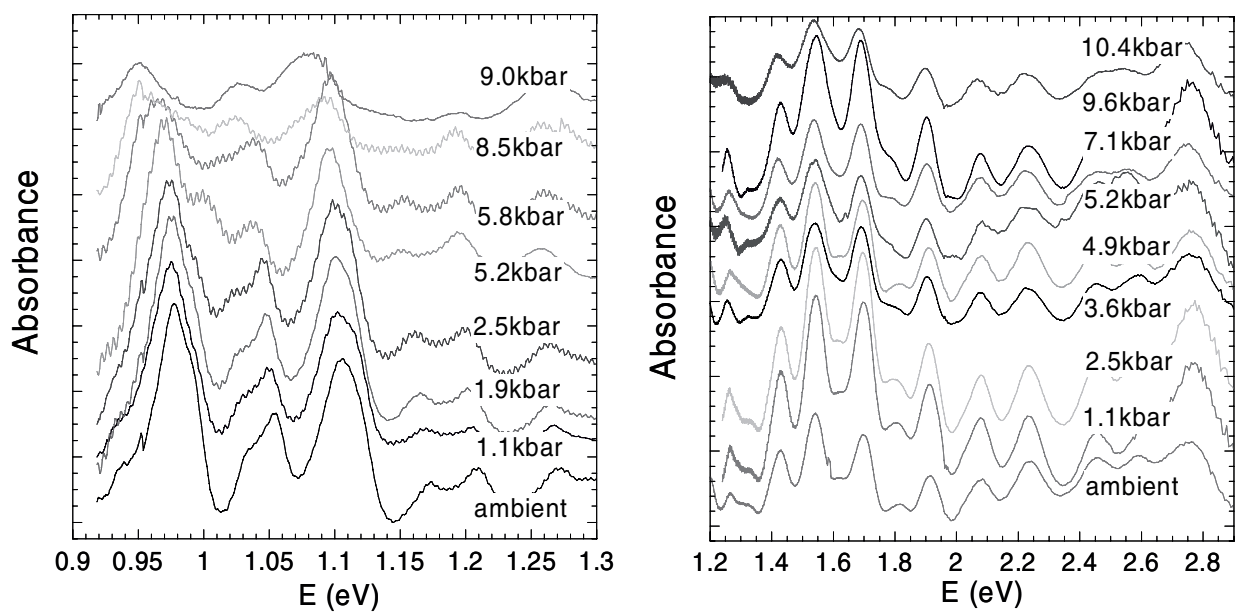

Fig. 2 Absorption spectra of Group A in the near infrared region (left panel) and absorption curves of Group B at higher energies (right panel) taken at different pressures. The spectral curves are vertically displaced for clarity. 
fringes between two diamond anvil facets. All peaks shift to lower energy with increasing hydrostatic pressure, opposite to most direct-gap semiconductors. The width, line-shape and intensity of all peaks are well maintained up to $8.5 \mathrm{kbar}$, at which the peaks start to weaken and broaden. At pressures above $\sim 11 \mathrm{kbar}$, the sample suspension turns from a homogenous liquid into a multi-grain-structured phase and the resultant light scattering severely reduces the signal intensity. An absorption spectrum taken at $15.6 \mathrm{kbar}$ shows no resolvable absorption features except a broad peak near $1.1 \mathrm{eV}$. This phase transition is related to the well-known crystallization of $\mathrm{D}_{2} \mathrm{O}$ into a tetragonal phase (water $\mathrm{VI}$ ) at $11 \mathrm{kbar}$ at room temperature [7]. In the crystallized phase of $\mathrm{D}_{2} \mathrm{O}$, the local non-hydrostatic component of pressure breaks the structural symmetry of the nanotubes and leads to a broadening of the electronic states associated with the optical transitions. The shift of the absorption peaks with hydrostatic pressure also cannot be attributed to the pressure response of the SDS- $\mathrm{D}_{2} \mathrm{O}$ solvent. Comparison of transmission spectra of a nanotube-free SDS- $\mathrm{D}_{2} \mathrm{O}$ suspension taken at ambient and at $\sim 5$ kbar pressure, respectively, shows only a small, wavelength-independent numerical change in background signal.

The absorption spectrum of Group B transitions under various hydrostatic pressures is shown in the right panel of Fig. 2. Unlike the Group A absorption peaks, some of the Group B peaks shift only slightly to lower energy, while some remain constant independent of the applied pressure. The line-shapes of all peaks remain unaffected within the hydrostatic pressure range.

The effect of hydrostatic pressure on the PL spectrum is shown in Fig. 3. The PL peaks also shift to lower energy under pressure. A rapid decrease in PL intensity was observed with increasing pressure. The intensity reduction is most likely caused by the non-radiative recombination centers induced on the surface of the nanotubes by the interaction with the surfactant coating under pressure.

Figure 4 shows the pressure coefficient obtained from a least-square linear fitting to the pressure dependence of each absorption peak. The numerical values of these pressure coefficients are listed in Table 1. The results indicate that, first of all, the application of hydrostatic pressure to SWNTs always decreases the bandgap $\left(E_{11}\right)$ irrespective of whether the value of $q$ is equal to 1 or 2 . This behavior is in contrast to the theoretical prediction [3] and the effects of uniaxial stress where both decrease and increase of the bandgap are observed depending on the value of $q[8,9]$. Secondly, the pressure coefficients for $E_{22}$ transitions are considerably smaller than those of $E_{11}$ transitions. The negative pressure coefficient is an intrinsic property of individual nanotubes, rather than an effect of inter-tube interactions or tube distortion within a bundle under high pressure.

The shift of the $E_{11}$ transition energy due to the pressure-induced change in volume of the SWNTs allows a direct estimation of the hydrostatic deformation potential for the band gap of the nanotubes. Because of the highly anisotropic geometry of nanotubes, their mechanical properties are different in the axial direction and the radial direction. It has been found that the linear modulus is insensitive to chirality for nanotubes with the same diameter [10]. A continuum model within classical elasticity theory was

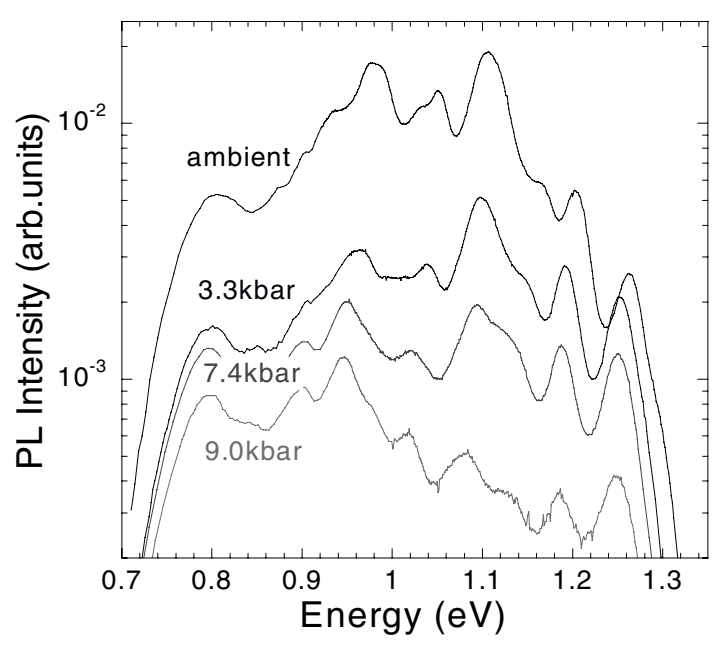

Fig. 3 Photoluminescence spectra of Group A recorded at selected pressures. 


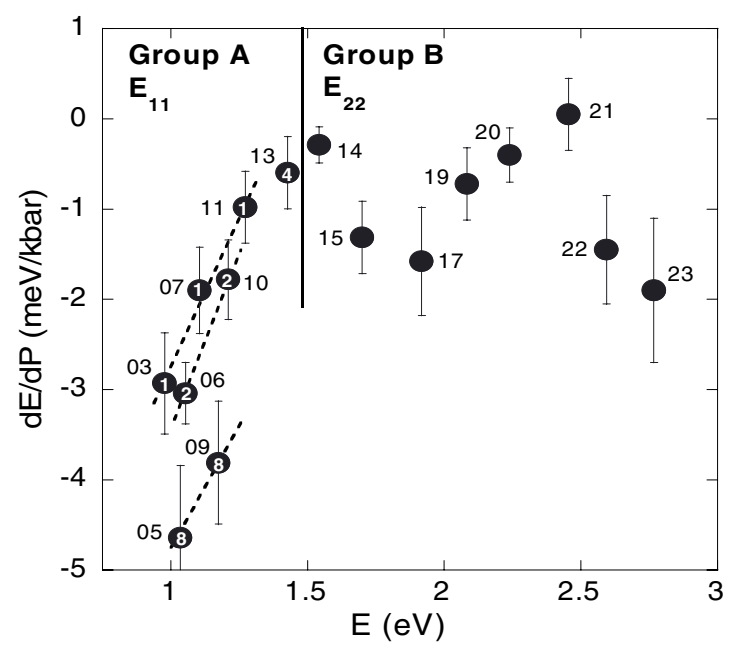

Fig. 4 Pressure coefficients as a function of peak energy. The numbers next to the data points are those marked the absorption peaks in Fig. 1. The numbers on the solid circles are the values of $n-m$ for $\mathrm{E}_{11}$ transitions. The dashed lines connect families with the same $n-m$ values.

introduced to model the bulk modulus $(\mathrm{d} P / \mathrm{d} \ln V)$ of SWNTs. The bulk modulus of individual nanotubes with diameter $d$ is given in the elastic model by [10]

$$
\frac{\mathrm{d} P}{\mathrm{~d} \ln V}=-\frac{4 Y}{1-2 v} \frac{w d}{(d+w)^{2}}\left[3+2 \frac{1+v}{1-2 v}\left(\frac{d-w}{d}\right)^{2}\right]^{-1},
$$

in which $Y \approx 1 \mathrm{TPa}$ is the Young's modulus, $v \approx 0.14$ is the Poisson's ratio, and $w=0.31 \mathrm{~nm}$ is the effective nanotube wall thickness. This equation yields a bulk modulus of $\sim 270 \mathrm{GPa}$ for $d=0.8 \mathrm{~nm}$. The hydrostatic deformation potential of the nanotube bandgap have been calculated from $\mathrm{d} E_{11} / \mathrm{d} \ln V=\left(\mathrm{d} E_{11} / \mathrm{d} P\right)(\mathrm{d} P / \mathrm{d} \ln V)$ and the calculated results are listed in Table 1 . The magnitude of deformation potentials is much smaller than that of diamond $(25 \mathrm{eV})$, but comparable to that of most other compound semiconductors.

Another striking effect shown in Fig. 4 is that the magnitude of pressure coefficients of Group A peaks $\left(\left|\mathrm{d} E_{11} / \mathrm{d} P\right|\right)$ tends to increase with decreasing transition energy. Since the main term in $E_{11}$ is inversely proportional to the tube diameter, this leads to the conclusion that the electronic states of largerdiameter nanotubes are more sensitive to hydrostatic pressure. In addition to this general trend, Fig. 4 also shows a pattern of larger $\left|\mathrm{d} E_{11} / \mathrm{d} P\right|$ for nanotubes with larger $n-m$ values. This pattern signifies a chiral angle $(\alpha)$ dependence in addition to a diameter $(d)$ dependence. We found that the pressure coefficient for all the $E_{11}$ data can be well described by the following equation,

$$
\frac{\mathrm{d} E_{11}}{\mathrm{~d} P}=a\left(d-d_{0}\right)+b \cos 3 \alpha
$$

where $a=-8.3 \mathrm{meV} /(\mathrm{kbar} \mathrm{nm}), b=-2.5 \mathrm{meV} / \mathrm{kbar}$ and $d_{0}=0.68 \mathrm{~nm} \mathrm{[11]} \mathrm{are} \mathrm{fitting} \mathrm{parameters.} \mathrm{The}$ first term, which is linear in diameter, may be related to the fact that the radial compression induced by external pressure is one phase of the nanotube's radial breathing vibrational phonon mode. This mode is experimentally visible in Raman spectra because it has a large resonant intensity enhancement as a result of its strong coupling to electronic excitations from the valence to the conduction bands [12]. The straight line in Fig. 5, where the pressure coefficients of various $E_{11}$ transitions are plotted as a function of $\cos 3 \alpha$, represents the dependence given by Eq. (3).

Tight binding calculation based on the grapheme $\pi$-electron model has shown that the band gap of a radially deformed SWNT should increase or decrease depending on the relative shift of the Fermi wave vector with respect to the nearest $\mathrm{K}$ line in the tube circumferential direction [13]. Therefore, the negative pressure coefficient of the bandgap is likely a higher-order effect beyond Eq. (3) that is based on the graphene $\pi$-electron model [14]. One possible mechanism that could account for the negative pressure 


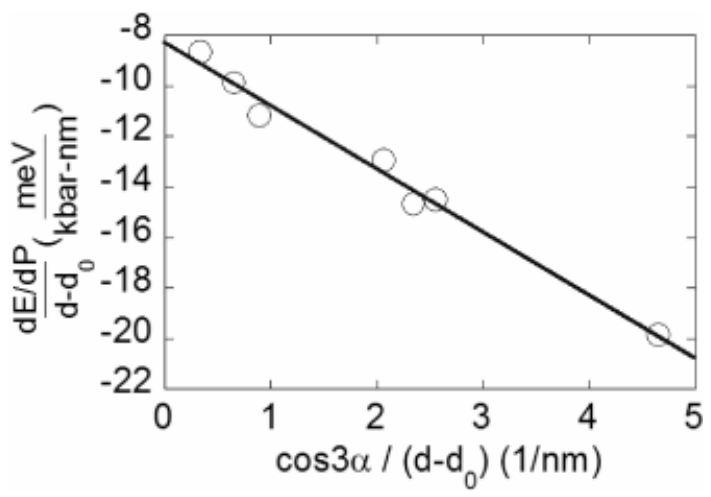

Fig. 5 The pressure coefficients of the $E_{11}$ transitions are scaled as a function of nanotube structure. The solid straight line represents the dependence given by Eq. (3).

coefficients is the $\sigma^{*}-\pi^{*}$ hybridization effect occurring near the large curvature points of nanotubes [15]. It has been demonstrated that the rehybridization between singlet $\sigma^{*}$ and $\pi^{*}$ conduction bands results in a drastic downward shift of the lowest conduction band in small diameter SWNTs [15] or radially deformed nanotubes [16]. The hybridization effect is the strongest in zigzag nanotubes where $\alpha=0$ and the weakest in armchair nanotubes where $\alpha=30^{\circ}$ [16-18]. The second lowest conduction band, which is responsible for the $E_{22}$ transitions, is less sensitive to the $\sigma^{*}-\pi^{*}$ hybridization [16]. These are consistent with the $\alpha$-dependence of $\left|\mathrm{d} E_{11} / \mathrm{d} P\right|$ in Eq. (3) and the insensitivity of the $E_{22}$ peaks to hydrostatic pressure, as shown in Fig. 4. A quantitative explanation of the observed negative hydrostatic pressure coefficients of SWNT bandgaps requires further theoretical exploration.

\section{Conclusions}

The effects of applied hydrostatic pressure on the optical transitions between the van Hove singularities above and below the Fermi level in individual singled-walled carbon nanotubes have been studied. We found that all singled-wall carbon nanotubes have negative hydrostatic pressure coefficient for the $E_{11}$ transition associated with the band-gap energy (the first van Hove transtion). The $E_{22}$ transitions associated with the excited confinement states (the second van Hove transtion) are found to have little pressure-induced energy shift. The experimental results show the numerical value of the pressure coefficient depends on the chirality for all nanotubes, along with a general trend of higher sensitivity of bandgap to hydrostatic pressure for larger-diameter nanotubes. The structure-dependent pressure coefficient for the band gap of the semiconducting nanotubes can be described using an empirical scaling equation in terms of nanotube diameter and chiral angle.

Acknowledgements This work at LBNL is supported by the Director, Office of Science, Office of Basic Energy Sciences, Division of Materials Sciences and Engineering, of the U.S. Department of Energy under Contract No. DE-AC03-76SF00098. R.B.W and S.M.B gratefully acknowledge the support from the National Science Foundation (Grant No. CHE-0314270) and the Welch Foundation (Grant No. C-0807).

\section{References}

[1] R. Saito, G. Dresselhaus, and M. S. Dresselhaus, Physical Properties of Carbon Nanotubes (Imperial College Press, Singapore, 1998).

[2] J. P. Salvetat, J. M. Bonard, N. H. Thomson, A. J. Kulik, L. Forro, W. Benoit, and L. Zuppiroli, Appl. Phys. A 69, 255 (1999).

[3] R. B. Capaz, C. D. Spataru, P. Tangney, M. L. Cohen, and S. G. Louie, phys. stat. sol. (b) 241, 3352 (2004) (this issue).

[4] M. J. O’Connell, S. M. Bachilo, C. B. Huffman, V. C. Moore, M. S. Strano, E. H. Haroz, K. L. Rialon, P. J. Boul, W. H. Noon, C. Kittrell, J. Ma, R. H. Hauge, R. B. Weisman, and R. E. Smalley, Science 297, 593 (2002).

[5] A. Hagen and T. Hertel, Nano Lett. 3, 383 (2003). 
[6] S. M. Bachilo, M. S. Strano, C. Kittrell, R. H. Hauge, R. E. Smalley, and R. B. Weisman, Science 298, 2361 (2002).

[7] W. F. Kuhs, J. L. Finney, C. Vettier, and D. V. Bliss, J. Chem. Phys. 81, 3612 (1984).

[8] E. D. Minot, Y. Yaish,V. Sazonova, J.-Y. Park, M. Brink, and P. L. McEuen, Phys. Rev. Lett. 90, 156401 (2003).

[9] J. Cao, Q. Wang, and H. Dai, Phys. Rev. Lett. 90, 157601 (2003).

[10] S. Reich, C. Thomsen, and P. Ordejon, Phys. Rev. B 65, 153407 (2002).

[11] J. Wu, W. Walukiewicz, W. Shan, E. Bourret-Courchesne, J. W. Ager, K. M. Yu, E. E. Haller, K. Kissell, S. M. Bachilo, R. B. Weisman, and R. E. Smalley, Phys. Rev. Lett. 93, 017404 (2004).

[12] M. A. Pimenta, A. Marucci, S. D. M. Brown, M. J. Matthews, A. M. Rao, P. C. Eklund, R. E. Smalley, G. Dresselhaus, and M. S. Dresselhaus, J. Mater. Res. 13, 2396 (1998).

[13] L. Yang and J. Han, Phys. Rev. Lett. 85, 154 (2000).

[14] R. Saito, G. Dresselhaus, and M. S. Dresselhaus, Phys. Rev. B 61, 2981 (2000).

[15] X. Blasé, L. X. Benedict, E. L. Shirley, and S. G. Louie, Phys. Rev. Lett. 72, 1878 (1994).

[16] J. C. Charlier, Ph. Lambin, and T. W. Ebbesen, Phys. Rev. B 54, 8377 (1996).

[17] A. Kleiner and S. Eggert, Phys. Rev. B 64, 113402 (2001).

[18] S. Reich, C. Thomsen, and P. Ordejon, Phys. Rev. B 65, 155411 (2002). 